\title{
Comparative Multifractal Detrended Fluctuation Analysis of Heavy Ion Interactions at a Few GeV to a Few Hundred GeV
}

\author{
Gopa Bhoumik, ${ }^{1}$ Argha Deb, ${ }^{1}$ Swarnapratim Bhattacharyya, ${ }^{2}$ and Dipak Ghosh ${ }^{1}$ \\ ${ }^{1}$ Nuclear and Particle Physics Research Centre, Department of Physics, Jadavpur University, Kolkata 700032, India \\ ${ }^{2}$ Department of Physics, New Alipore College, L Block, New Alipore, Kolkata 700053, India \\ Correspondence should be addressed to Gopa Bhoumik; ppgopa@gmail.com
}

Received 4 February 2016; Accepted 7 April 2016

Academic Editor: Ming Liu

Copyright (c) 2016 Gopa Bhoumik et al. This is an open access article distributed under the Creative Commons Attribution License, which permits unrestricted use, distribution, and reproduction in any medium, provided the original work is properly cited. The publication of this article was funded by $\mathrm{SCOAP}^{3}$.

We have studied the multifractality of pion emission process in ${ }^{16} \mathrm{O}-\mathrm{AgBr}$ interactions at $2.1 \mathrm{AGeV}$ and $60 \mathrm{AGeV}$, ${ }^{12} \mathrm{C}$ $\mathrm{AgBr}$ and ${ }^{24} \mathrm{Mg}-\mathrm{AgBr}$ interactions at $4.5 \mathrm{AGeV}$, and ${ }^{32} \mathrm{~S}-\mathrm{AgBr}$ interactions at $200 \mathrm{AGeV}$ using Multifractal Detrended Fluctuation Analysis (MFDFA) method which is capable of extracting the actual multifractal property filtering out the average trend of fluctuation. The analysis reveals that the pseudorapidity distribution of the shower particles is multifractal in nature for all the interactions; that is, pion production mechanism has inbuilt multiscale self-similarity property. We have employed MFDFA method for randomly generated events for ${ }^{32} \mathrm{~S}-\mathrm{AgBr}$ interactions at $200 \mathrm{AGeV}$. Comparison of expt. results with those obtained from randomly generated data set reveals that the source of multifractality in our data is the presence of long range correlation. Comparing the results obtained from different interactions, it may be concluded that strength of multifractality decreases with projectile mass for the same projectile energy and for a particular projectile it increases with energy. The values of ordinary Hurst exponent suggest that there is long range correlation present in our data for all the interactions.

\section{Introduction}

The study of correlation and fractality is an active area of research in many fields including heavy ion collisions [15]. Natural systems, which have irregular pattern at different scales, exhibit fractal nature. Fractals are generally classified into two categories: (i) monofractal and (ii) multifractals. For monofractals scaling properties of the system are identical throughout the system; on the other hand, "multifractals" are more complicated self-similar structure that consist of a number of weighted fractals with different noninteger dimensions. As the scaling properties are dissimilar in different parts of the system, multifractal systems require at least more than one scaling exponent to describe the scaling behavior of the system [6]. Investigation of multifractality is of great importance as its origin may be associated with the presence of long range correlation in the system. Correlation study has the potential to provide information about the characteristics of system evolution. Moreover, multifractal analysis is effective in understanding the underlying dynamics of any complex system such as pionisation in high energy nucleusnucleus interactions. To get both qualitative and quantitative idea concerning the multiparticle production mechanism [7] multifractal analysis is expected to be very fruitful. Such a behavior has been observed for vast majority of high energy multiparticle production experiments [8-10].

The investigation of fractal dimension in hadronic multiparticle production was carried out probably for the first time by Carruthers and Duong-Van [11]. But there was no formalism developed for a systematic fractal study. A systematic approach for the fractal study was suggested by Hwa ( $G_{q}$ moment) [12]. But those $G_{q}$ moments are found to be influenced by statistical fluctuations especially for the low multiplicity events. Later in order to avoid large statistical fluctuations and exclude low multiplicity events Hwa and Pan [13] proposed a modified $G_{q}$ moment method introducing a step function, which acts as a filter to the low multiplicity events [14-16]. Also these modified $G_{q}$ moments suffer from 
the demerit that they are defined only for positive orders $(q)$ and hence they are unable to explore the whole multifractal spectrum. Afterwards, a number of techniques [17-20] were developed for the fractal study of multiparticle data. The techniques developed by Hwa [12] and Takagi [17] are the most popular and have been used in many cases $[2,21-$ $23]$ to analyze multipion production process. However, none of these methods can disentangle the dynamical "signal" from the "background." Trending behaviors usually give rise to spurious multifractal effects for the analyzed series. Therefore, it is essential to study the intrinsic fluctuations characterizing the dynamical process after filtering out the average trending behavior.

Sophisticated methods have been invented to characterize the actual fluctuations extracted from the average behavior and the fractal nature of nonstationary time series. These include detrended fluctuation analysis (DFA) and its variance $[24,25]$, the wavelet transform [26, 27] based multiresolution analysis [28, 29], and Multifractal Detrended Fluctuation Analysis (MFDFA) [30]. DFA technique [24] was developed in order to determine minutely the presence of any long range correlation $[24,31]$ in a nonstationary series. However, despite a multitude of real-data analyses, a proper detection of the multifractality in the experimental data still presents much difficulty and is not always reliable [32]. MFDFA technique [30] is actually a generalization of standard DFA technique for the characterization of multifractal nature of a series. One main reason to employ MFDFA method is to avoid fallacious detection of correlations leading to multifractality which are artifacts evolving due to the nonstationarity of the signal. Thus, MFDFA is a powerful technique which has been applied successfully to characterize fluctuation in a variety of fields like finance [33-36], medicine [37, 38], natural science $[39,40]$, solid state physics [41, 42], and so forth.

The spectrum of references of application of MFDFA technique is not a complete one. Recently, MFDFA method has been applied to analyze the pseudorapidity and azimuthal angle distribution of the pions produced in $\mathrm{Au}+\mathrm{Au}$ interactions at $200 \mathrm{GeV} /$ nucleon by Zhang et al. [43]. They studied a sample of only 10 events. Another group, Wang and Yang [44], studied the same interactions for UrQMD generated data using the same method. The DFA and MFDFA methods are also used by Mali et al. [45] to characterize the particle density fluctuation for ${ }^{28} \mathrm{Si}+\mathrm{Ag} / \mathrm{Br}$ interactions at $14.5 \mathrm{GeV} /$ nucleon and ${ }^{32} \mathrm{~S}+\mathrm{Ag} / \mathrm{Br}$ interactions at $200 \mathrm{GeV} /$ nucleon. These analyses [43-45] suggest that the MFDFA approach is a reasonably good technique for the multifractal analysis of multiparticle production process in high energy nucleusnucleus (A-A) interactions and hence should be applied for understanding the dynamics of the process.

In this paper we have studied the pseudorapidity distribution of the pions produced in ${ }^{16} \mathrm{O}-\mathrm{AgBr}$ interactions at $2.1 \mathrm{AGeV}$ and $60 \mathrm{AGeV},{ }^{12} \mathrm{C}-\mathrm{AgBr}$ and ${ }^{24} \mathrm{Mg}$ - $\mathrm{AgBr}$ interactions at $4.5 \mathrm{AGeV}$, and ${ }^{32} \mathrm{~S}-\mathrm{AgBr}$ interactions at $200 \mathrm{AGeV}$ in the framework of MFDFA. The analysis is expected to reveal a comparative view of the genuine multifractal parameters for interactions initiated by projectiles of various energies and masses.

\section{Experimental Section}

The present analysis is performed on the interactions of ${ }^{16} \mathrm{O}$ beam at $2.1 \mathrm{AGeV}$ and $60 \mathrm{AGeV},{ }^{12} \mathrm{C}$ beam and ${ }^{24} \mathrm{Mg}$ both at $4.5 \mathrm{AGeV}$, and ${ }^{32} \mathrm{~S}$ beam at $200 \mathrm{AGeV}$ with $\mathrm{AgBr}$ being the target present in nuclear emulsion.

ILFORD G5 nuclear photographic emulsion plates were irradiated horizontally with a beam of ${ }^{16} \mathrm{O}$ nuclei at energy of $2.1 \mathrm{AGeV}$ obtained from BEVALAC Berkley [46]. The data for ${ }^{12} \mathrm{C}-\mathrm{AgBr}$ [47] and ${ }^{24} \mathrm{Mg}-\mathrm{AgBr}$ [48] interactions were obtained by exposing NIKFI BR2 emulsion plates to the beams of ${ }^{12} \mathrm{C}$ and ${ }^{24} \mathrm{Mg}$ nucleus, of energy $4.5 \mathrm{AGeV}$ at JINR, Dubna, Russia. The data of ${ }^{16} \mathrm{O}-\mathrm{AgBr}$ interactions at $60 \mathrm{AGeV}$ and ${ }^{32} \mathrm{~S}-\mathrm{AgBr}$ interactions at $200 \mathrm{AGeV}$ were obtained by exposing the stacks of ILFORD G5 emulsion plates to a beam of ${ }^{16} \mathrm{O}$ nucleus of energy $60 \mathrm{AGeV}$ and ${ }^{32} \mathrm{~S}$ nucleus of energy $200 \mathrm{AGeV}$, respectively, using the super proton synchrotron (SPS) at CERN [49]. A Leitz Ortholux microscope with a 10x objective and 25x ocular lens provided with a Brower travelling stage was used to scan the plates of ${ }^{16} \mathrm{O}-\mathrm{AgBr}$ interactions at $2.1 \mathrm{AGeV}$. A Leitz Metalloplan microscope with a 10x objective and 10x ocular lens provided with a semiautomatic scanning stage has been used to scan the other four interaction plates. Two observers scanned each plate independently so that the biases in detection, counting, and measurement could be minimized and consequently the scanning efficiency could be increased. After finding a primary interaction induced by the incoming projectile, the number of secondary tracks in an event belonging to each category was counted using oil immersion objectives. Measurements were carried out with the help of an oil immersion objective of 100x magnification. The measuring system fitted with both the microscopes has $1 \mu \mathrm{m}$ resolution along the $x$ axis and $y$-axis and $0.5 \mu \mathrm{m}$ resolution along the $z$-axis.

Events were chosen according to the criteria given below.

(a) The incident beam track would have to lie within $3^{\circ}$ from main beam direction. (b) Events that occurred within $20 \mu \mathrm{m}$ from the top and bottom surfaces of the pellicle were rejected. (c) All the primary beam tracks were followed in the backward direction to ensure that the events chosen did not include interactions from the secondary tracks of the other interactions.

According to nuclear emulsion terminology [50], particles emitted after interactions can be classified as the shower, grey, and black particles. Shower particles are mostly (about more than 90\%) due to pions with a small admixture of $K$-mesons and hyperons having ionization $I \leq 1.4 I_{0}$ and velocity greater than $0.7 c$, where $I_{0}$ is the minimum ionization of a singly charged particle produced in the emulsion medium. Grey particles are mainly fast target recoil protons with energies up to $400 \mathrm{MeV}$. They have ionization $1.4 I_{0} \leq$ $I<10 I_{0}$. They have velocities lying between $0.3 c$ and $0.7 c$. Black particles consist of both singly and multiply charged fragments of the target nucleus with ionization $I \geq 10 I_{0}$ and velocity less than $0.3 c$.

Along with the above stated three kinds of particle, there could also be a few projectile fragments. These projectile 
fragments are the spectator parts of the incident projectile nuclei that do not directly participate in an interaction.

In the experiments with the nuclear emulsion track detectors, interactions may be with three different types of targets, for example, hydrogen $(\mathrm{H})$, light nuclei $(\mathrm{CNO})$, and heavy nuclei $(\mathrm{AgBr})$, present in the emulsion medium. Events, with $N_{h} \leq 1$, occur because of the collision between hydrogen and the projectile beam. Events with $2 \leq N_{h} \leq 8$ are due to collisions of projectile with light nuclei and events with $N_{h}>8$ are due to collisions with heavy nuclei. Here $N_{h}$, the number of heavy tracks, is the total number of black and grey tracks. In our study, only events having a number of heavy tracks greater than $8\left(N_{h}>8\right)$ have been selected to exclude the $\mathrm{H}$ and $\mathrm{CNO}$ events.

According to the selection procedure mentioned above, we have chosen 730 events of ${ }^{16} \mathrm{O}-\mathrm{AgBr}$ interactions at $2.1 \mathrm{AGeV}$ [46], 800 events of ${ }^{12} \mathrm{C}-\mathrm{AgBr}$ [51] and ${ }^{24} \mathrm{Mg}$ $\mathrm{AgBr}$ [52] interactions at $4.5 \mathrm{AGeV}, 250$ events of ${ }^{16} \mathrm{O}-\mathrm{AgBr}$ interactions at $60 \mathrm{AGeV}$ [53], and 140 events of ${ }^{32} \mathrm{~S}-\mathrm{AgBr}$ interactions at $200 \mathrm{AGeV}$ [54]. The present analysis has been performed on the pion tracks only. The emission angle $(\theta)$ was measured for each pion track with respect to the beam direction by taking readings of the coordinates of the interaction point $\left(X_{0}, Y_{0}, Z_{0}\right)$, coordinates $\left(X_{1}, Y_{1}, Z_{1}\right)$ of a point at some distance away from the interaction point on each secondary track, and coordinates $\left(X_{i}, Y_{i}, Z_{i}\right)$ of a point on the incident beam. From " $\theta$ " the pseudorapidity variable $(\eta=-\ln \tan (\theta / 2))$, which may be treated as a convenient substitute of the rapidity variable of a particle when the rest mass of the particle can be neglected in comparison to its energy or momentum, was calculated for each pion track.

\section{MFDFA Method}

The MFDFA technique was developed by Kantelhardt et al. [30] as a generalization of standard DFA method to analyze nonstationary time series. If $\left\{x_{k}, k=1,2,3, \ldots, N\right\}$ is the signal of length $N$, MFDFA consists of the following steps [30] among which the first three steps include the ordinary DFA technique.

Step 1. Calculation of the signal profile, which is the cumulative sum of the signal to be analyzed, is according to

$$
y(j)=\sum_{k=1}^{j}\left[x_{k}-\langle x\rangle\right], \quad \text { where } j \text { runs from } 1 \text { to } N .
$$

Step 2. Consider division of the profile $y(j)$ into $N_{s}=$ $\operatorname{int}(N / s)$ numbers of nonoverlapping segments of length $s$. Since $N$ is not an integer multiple of $s$, a small part of the signal will be left at the end. In order to include that part, the same process is repeated starting from the other end. Thus, one obtains altogether $2 N_{s}$ number of segments.

Step 3. Consider determination of the local trend associated with each of such $2 N_{s}$ segments by a least square polynomial fit of the series in a particular segment, as well as calculation of the variance of the series. The variance of the series relative to the local trend in a given segment $v$ of length $s$ can be calculated as

$$
F^{2}(\nu, s)=\frac{1}{s} \sum_{j=1}^{s}\left\{y[(\nu-1) s+j]-y_{\text {fit }}^{l v}(j)\right\}^{2},
$$

where $y_{\text {fit }}^{l v}(j)$ is the fitting polynomial of order $l$ for the $\nu$ th segment.

Step 4. qth order fluctuation function $F_{q}(s)$ is then given by [32]

$$
\begin{aligned}
F_{q}(s) & =\left\{\frac{1}{2 N_{s}} \sum_{\gamma=1}^{2 N_{s}}\left[F^{2}(\nu, s)\right]^{q / 2}\right\}^{1 / q} \quad \text { for } q \neq 0 \\
& =\exp \left\{\frac{1}{4 N_{s}} \sum_{\nu=1}^{2 N_{s}} \ln F(\nu, s)\right\} \quad \text { for } q=0 .
\end{aligned}
$$

Step 5. To investigate the scaling behavior, one has to calculate $F_{q}(s)$ for various $s$ values. It should be remembered that the sample size of the smallest segment (or scale) should be much larger compared to the polynomial order $l$ in order to prevent an overfitted trend.

3.1. Scaling Behavior and Multifractality. Presence of any long range correlation in the system will appear in the multifractal behavior of the system and then $F_{q}(s)$ will show a power law dependence on scale $s$ like

$$
F_{q}(s) \propto s^{h(q)} .
$$

The scaling exponent $h(q)$ is known as generalized Hurst exponent. For $q=2$ it reduces to the ordinary Hurst exponent $H$ [55]. $H \neq 0.5$ indicates the presence of long range correlation in the system. An exponent $H>0.5$ corresponds to positive correlation in the system and $H<$ 0.5 corresponds to the presence of anticorrelation in the system. For a monofractal series $h(q)$ is independent of $q$ and the fluctuation function $F_{q}(s)$ is similar for all subsignals. On the other hand, if large and small fluctuations scale differently then there will be a notable dependence of $h(q)$ on $q$. Particularly for multifractal series $h(q)$ decreases with order $q$; for +ve $q$ values $h(q)$ describes the scaling behavior of the segments with large fluctuations and for -ve $q$ 's it describes the scaling behavior of the segments with small fluctuations.

The multifractal exponent $\tau(q)$ can be derived from $h(q)$ $[30,55]$ using (6). Consider

$$
\tau(q)=q h(q)-1
$$

The singularity spectrum $f(\alpha)$ can be obtained from $\tau(q)$ through a Legendre transform $[20,55,56]$ :

$$
f(\alpha)=q \alpha-\tau(q), \quad \text { where } \alpha=\frac{\partial \tau(q)}{\partial q} .
$$

The width of the multifractal spectrum is the difference between the maximum and minimum value of $\alpha$; that is,

$$
\text { width of spectrum }=\alpha_{\max }-\alpha_{\min } .
$$


It has been proposed by Ashkenazy et al. [57] and Shimizu et al. [58] that the width of a multifractal spectrum is a measure of the degree of multifractality. The broader the spectrum, the richer the multifractality [59].

Though originally MFDFA technique was developed for nonstationary time series analysis, it can also be used equally for nonuniform distribution like rapidity distribution of high energy multiparticle production process [43-45]. In principle, multifractality of a natural system may originate either (a) due to broad probability density function (nonGaussian distribution) of the concerned parameter or (b) due to the simultaneous presence of dissimilar characteristics of long range correlations for large as well as small fluctuations or sometimes (c) due to both reasons (a) and (b) [60]. MFDFA technique allows one to identify the source of multifractality when it is applied to the randomly shuffled distribution of the same. For shuffled distribution though the probability distribution function remains unchanged, all possible correlations are wiped out from the distribution. Thus, if multifractality is originated from type (b), then randomly generated distribution will show an arbitrary behavior with $h(q)_{\text {random }}=0.5$. On the other hand, if the source of multifractality is type (a), then for the generated distribution generalized Hurst exponent will be identical to that of the original one $\left(h(q)_{\text {random }}=h(q)_{\text {experimental }}\right)$. Furthermore, for reason (c) the generated series will show a weaker multifractality than that of the experimental one.

\section{Results and Discussions}

In the current analysis we have focused on ${ }^{16} \mathrm{O}-\mathrm{AgBr}$ interactions at $2.1 \mathrm{AGeV}$ and $60 \mathrm{AGeV},{ }^{12} \mathrm{C}-\mathrm{AgBr}$ and ${ }^{24} \mathrm{Mg}$ $\mathrm{AgBr}$ interactions at $4.5 \mathrm{AGeV}$, and ${ }^{32} \mathrm{~S}-\mathrm{AgBr}$ interactions at $200 \mathrm{AGeV}$. For each interaction we have considered only those events which have multiplicities greater than average multiplicity of the corresponding interaction. Pseudorapidity distribution of the pions produced in each such event was obtained for 0.1 rapidity interval. MFDFA technique as discussed has been utilized to analyze the pseudorapidity distribution of the shower particles corresponding to each selected event. Here, the term scale $(s)$ corresponds to the width of each segment in the pseudorapidity space. The smallest scale is so chosen that at least 10 data points are within it; that is, it covers one pseudorapidity unit in the pseudorapidity space. The fluctuation function $F_{q}(s)$ has been calculated for different " $s$ " values with " $q$ " varying from -5 to +5 with integer values only using (3) for each selected event. Event average of fluctuation function $\left(\left\langle F_{q}(s)\right\rangle\right)$ is obtained by averaging over all selected events. The plot of $\log \left\langle F_{q}(s)\right\rangle$ with $\log (s)$ for $q=+5,0$, and -5 is shown in Figure 1 for all interactions. For any particular order $q$ the event average fluctuation function $\left\langle F_{q}(s)\right\rangle$ manifests power law dependence on $s$ and consequently all the plots are of straight line nature with different slope. The point symbols in Figure 1 represent the values of $\log \left\langle F_{q}(s)\right\rangle$ for different $\log (s)$ whereas the solid lines represent the regression fit of the same. $\left\langle F_{q}(s)\right\rangle$ for different " $q$ " values shows a convergent trend as the scale increases. The slope for $\operatorname{linear}$ fit of $\log \left\langle F_{q}(s)\right\rangle$ versus $\log (s)$
TABLE 1: Hurst exponent for the different interactions.

\begin{tabular}{lc}
\hline Interactions & $\begin{array}{c}\text { Value } h_{q} \text { at } \\
q=2\end{array}$ \\
\hline${ }^{12} \mathrm{C}-\mathrm{AgBr}$ interactions at $4.5 \mathrm{AGeV}$ & 0.803 \\
${ }^{16} \mathrm{O}-\mathrm{AgBr}$ interactions at $2.1 \mathrm{AGeV}$ & 0.922 \\
${ }^{16} \mathrm{O}-\mathrm{AgBr}$ interactions at $60 \mathrm{AGeV}$ & 1.352 \\
${ }^{24} \mathrm{Mg}-\mathrm{AgBr}$ interactions at $4.5 \mathrm{AGeV}$ & 0.801 \\
${ }^{32} \mathrm{~S}-\mathrm{AgBr}$ interactions at $200 \mathrm{AGeV}$ & 1.571 \\
Shuffled ${ }^{32} \mathrm{~S}-\mathrm{AgBr}$ interactions at $200 \mathrm{AGeV}$ & 0.511 \\
\hline
\end{tabular}

graph provides the generalized Hurst exponent $h(q) . h(q)$ values vary for different $q$ 's.

The variation of $h(q)$ with $q$ has been depicted in Figure 2. For all the interactions $h(q)$ is $q$ dependent and it decreases with $q$, signifying multifractal nature of the rapidity distribution as manifested by the different scaling behaviors for large and small fluctuations. $h(q)$ values for $q<0$ seem to be higher than those for $q>0$. This typical feature of $h(q)$ is consistent with the observation by Manimaran et al. [29] for multifractal time series. The ordinary Hurst exponent $H$, that is, $h(q)$ for $q=2$, is given in Table 1 for all the interactions. $H$, as mentioned earlier, gives an idea about the correlation present in the system. For all the considered interactions $H>0.5$ indicates that long range correlation exists in our data for all the interactions.

We have also calculated $\tau(q)$ for different $q$ values according to (5). The plot of $\tau(q)$ against $q$ is shown in Figure 3. The nonlinear variation of $\tau(q)$ with $q$ also confirms the fact that the pion density fluctuation in the rapidity space is multifractal in nature; that is, pion production mechanism has an inbuilt multiscale self-similarity property.

In order to shed light on the possible origin of the demonstrated multifractal behavior we have randomly shuffled the rapidity distribution of each selected event for all interactions and have employed MFDFA method to the shuffled data using the same approach as before. Results were found to be identical for all the interactions. Here we present the result of comparison of experimental and shuffled data only for ${ }^{32} \mathrm{~S}$ $\mathrm{AgBr}$ interactions at $200 \mathrm{AGeV}$.

Figure 4 shows the plot of $h_{q \text {,shuffle }}$ with $q$ along with $h_{q, \text { expt. }}$ versus $q . h_{q, \text { shuffle }}$ values remain around 0.5 (average $0.56)$ with a minor dependence on $q$ with $h_{q}(q=2)=$ 0.51 . $H \approx 0.5$ suggests that multifractality in our data is mainly sourced from the presence of long range correlation in the data. $\tau(q)$ versus $q$ plot (Figure 5) for the shuffled and experimental data further strengthens this fact. $\tau(q)_{\text {shuffle }}$ shows an almost linear behavior instead of nonlinear one as for the experimental data. Similar behaviors were observed for the other interactions also.

To have a quantitative idea about the multifractality we have computed the multifractal spectrum corresponding to each interaction. The multifractal spectrums corresponding to different interactions have been displayed in Figure 6. Figure 7 shows the spectrum for ${ }^{32} \mathrm{~S}-\mathrm{AgBr}$ interactions for both expt. and shuffled distributions. For shuffled data spectrum has negligible width $(\alpha=0.38)$ with respect to experimental 
TABLE 2: Width of multifractal spectrum for different interactions.

\begin{tabular}{llll}
\hline Interactions & $\alpha_{\max }$ & $\alpha_{\min }$ & Width of spectrum \\
\hline${ }^{12} \mathrm{C}-\mathrm{AgBr}$ interactions at $4.5 \mathrm{AGeV}$ & 2.15 & 0.49 & 1.66 \\
${ }^{16} \mathrm{O}-\mathrm{AgBr}$ interactions at $2.1 \mathrm{AGeV}$ & 2.04 & 0.60 & 1.44 \\
${ }^{16} \mathrm{O}-\mathrm{AgBr}$ interactions at $60 \mathrm{AGeV}$ & 4.34 & 0.87 & 3.47 \\
${ }^{24} \mathrm{Mg}-\mathrm{AgBr}$ interactions at $4.5 \mathrm{AGeV}$ & 1.50 & 0.58 & 0.92 \\
${ }^{32} \mathrm{~S}-\mathrm{AgBr}$ interactions at $200 \mathrm{AGeV}$ & 3.51 & 1.12 & 2.39 \\
Shuffled ${ }^{32} \mathrm{~S}-\mathrm{AgBr}$ interactions at $200 \mathrm{AGeV}$ & 0.76 & 0.38 & 0.38 \\
\hline
\end{tabular}

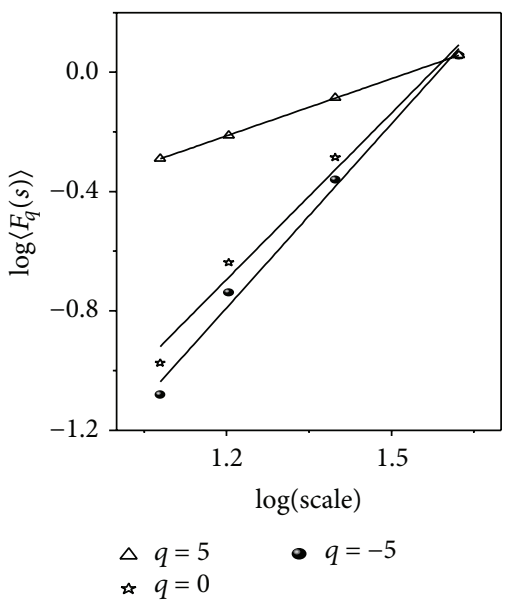

(a)

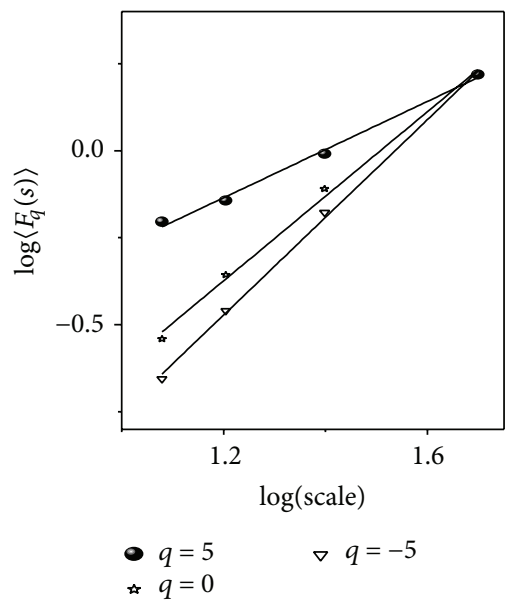

(b)

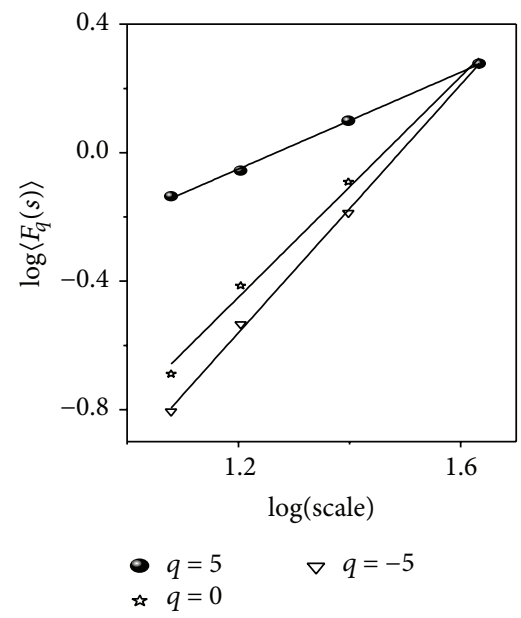

(c)

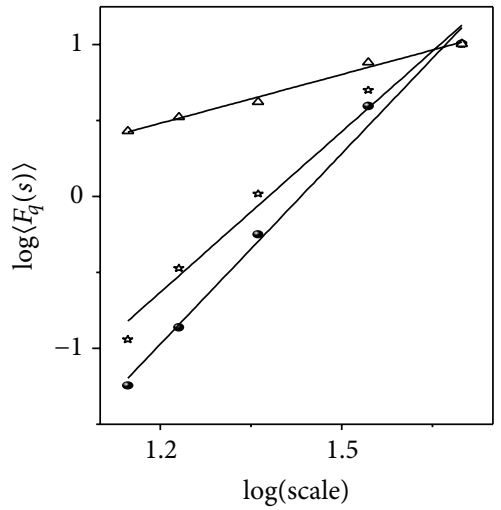

$$
\Delta q=5 \quad \bullet q=-5
$$$$
\nLeftarrow q=0
$$

(d)

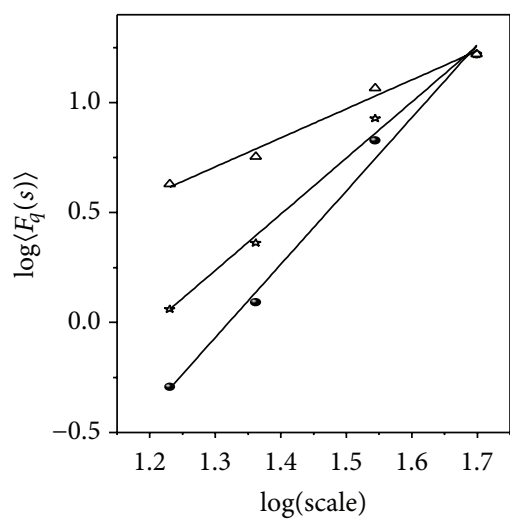

$\triangle q=5 \quad \bullet q=-5$

« $q=0$

(e)

Figure 1: Plot of logarithm of fluctuation function with logarithm of scale for (a) ${ }^{12} \mathrm{C}-\mathrm{AgBr}$ interactions at $4.5 \mathrm{AGeV}$, (b) ${ }^{24} \mathrm{Mg}$ - $\mathrm{AgBr}$ interactions at $4.5 \mathrm{AGeV}$, (c) ${ }^{16} \mathrm{O}-\mathrm{AgBr}$ interactions at $2.1 \mathrm{AGeV}$, (d) ${ }^{16} \mathrm{O}-\mathrm{AgBr}$ interactions at $60 \mathrm{AGeV}$, and (e) ${ }^{32} \mathrm{~S}-\mathrm{AgBr}$ interactions at $200 \mathrm{AGeV}$.

data $(\alpha=2.39)$ and also the spectrum shifted to the left of the experimental spectrum as expected when multifractality is mainly sourced [60] from type (b) cause as discussed earlier.

To compare the strengths of multifractality, we have calculated the width of the multifractal spectrum for each of the interactions. These values have been presented in Table 2 . The spectrum width is found to vary for different interactions suggesting that the strength of multifractality depends upon the mass and energy of the projectile beam. The spectrum width values reveal the following notable features of the heavy ion interaction process:

(i) Comparison of ${ }^{16} \mathrm{O}-\mathrm{AgBr}$ interactions at $2.1 \mathrm{AGeV}$ and at $60 \mathrm{AGeV}$ indicates that, for a particular projectile, strength of multifractality increases with the energy of the projectile.

(ii) From ${ }^{24} \mathrm{Mg}-\mathrm{AgBr}$ interactions and ${ }^{12} \mathrm{C}-\mathrm{AgBr}$ interactions at the same energy $4.5 \mathrm{AGeV}$, it is evident 


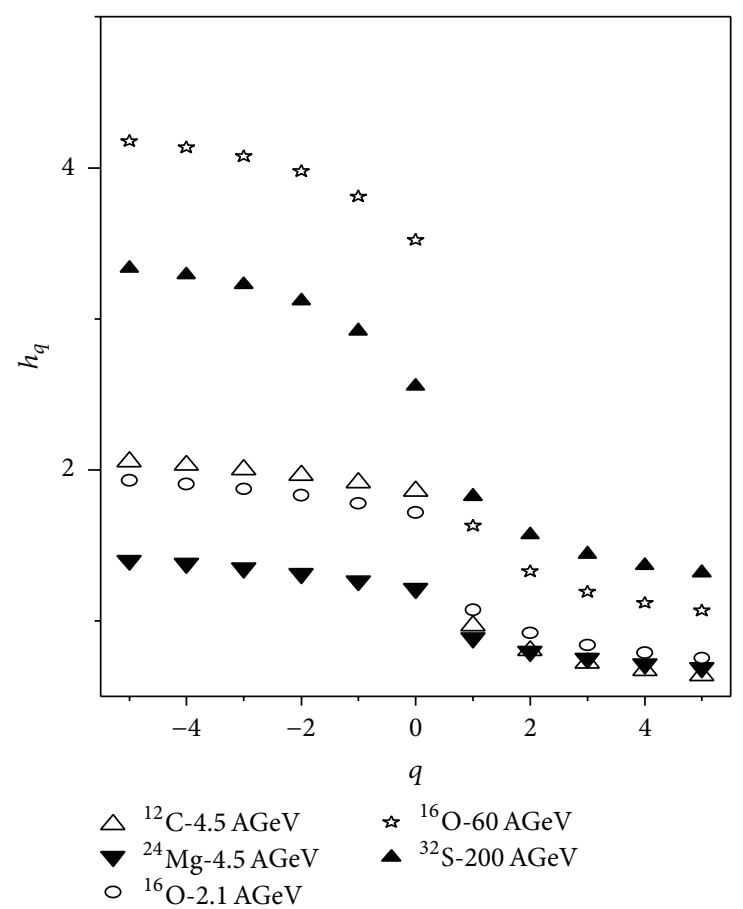

FIGURE 2: Plot of variation of generalized Hurst exponent $\left(h_{q}\right)$ with $\operatorname{order}(q)$ for all the interactions.

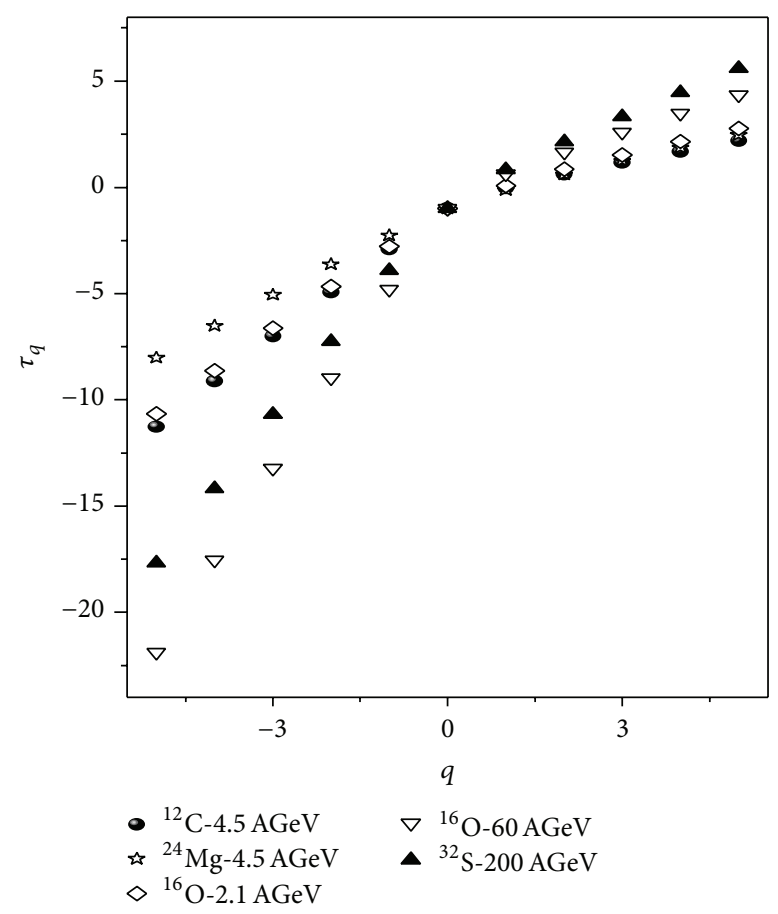

FIGURE 3: Plot of $\tau(q)$ versus $q$ for different interactions.

that the strength of multifractality decreases with projectile mass.

(iii) For projectiles with different mass and energies, multifractality decreases if there are simultaneous decrease in energy and increase in mass of the projectile beam (e.g., the pair of interactions (i)

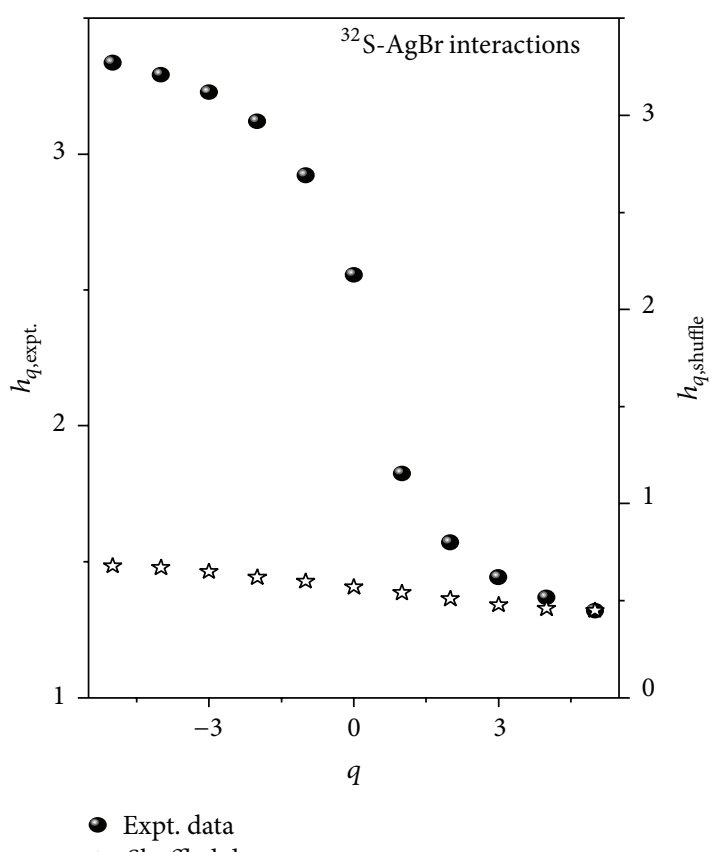

is Shuffled data

FIGURE 4: Comparison of ordinary Hurst exponent for experimental and shuffled data for ${ }^{32} \mathrm{~S}-\mathrm{AgBr}$ interactions at $200 \mathrm{AGeV}$.

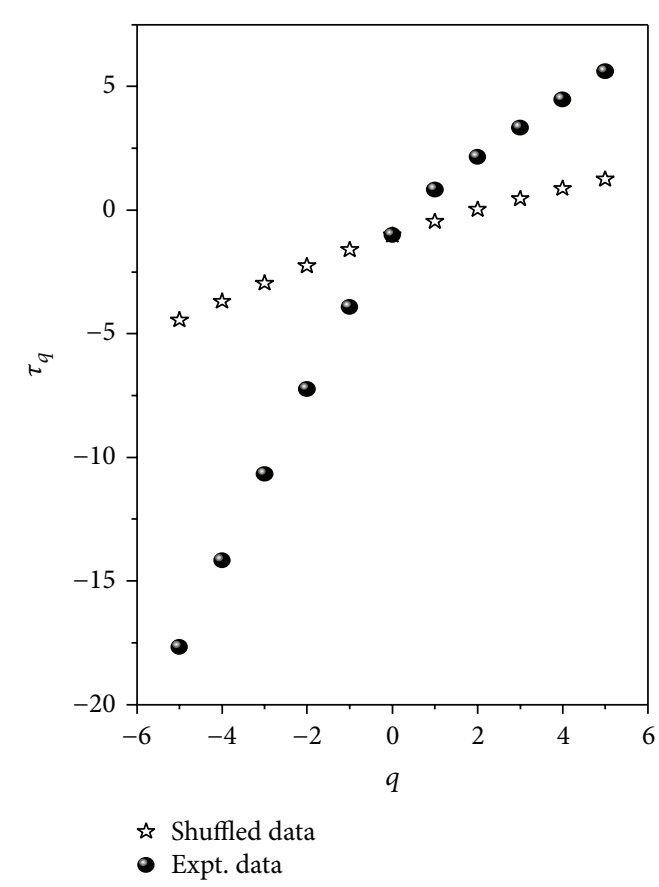

FIGURE 5: Comparison of $\tau_{q}$ spectra for experimental and shuffled data for ${ }^{32} \mathrm{~S}-\mathrm{AgBr}$ interactions at $200 \mathrm{AGeV}$.

${ }^{16} \mathrm{O}-\mathrm{AgBr}$ at $60 \mathrm{AGeV}$ and ${ }^{24} \mathrm{Mg}-\mathrm{AgBr}$ at $4.5 \mathrm{AGeV}$ and (ii) ${ }^{12} \mathrm{C}-\mathrm{AgBr}$ at $4.5 \mathrm{AGeV}$ and ${ }^{16} \mathrm{O}-\mathrm{AgBr}$ at $2.1 \mathrm{AGeV}$ ). This observation agrees very well with the previous two observations. 


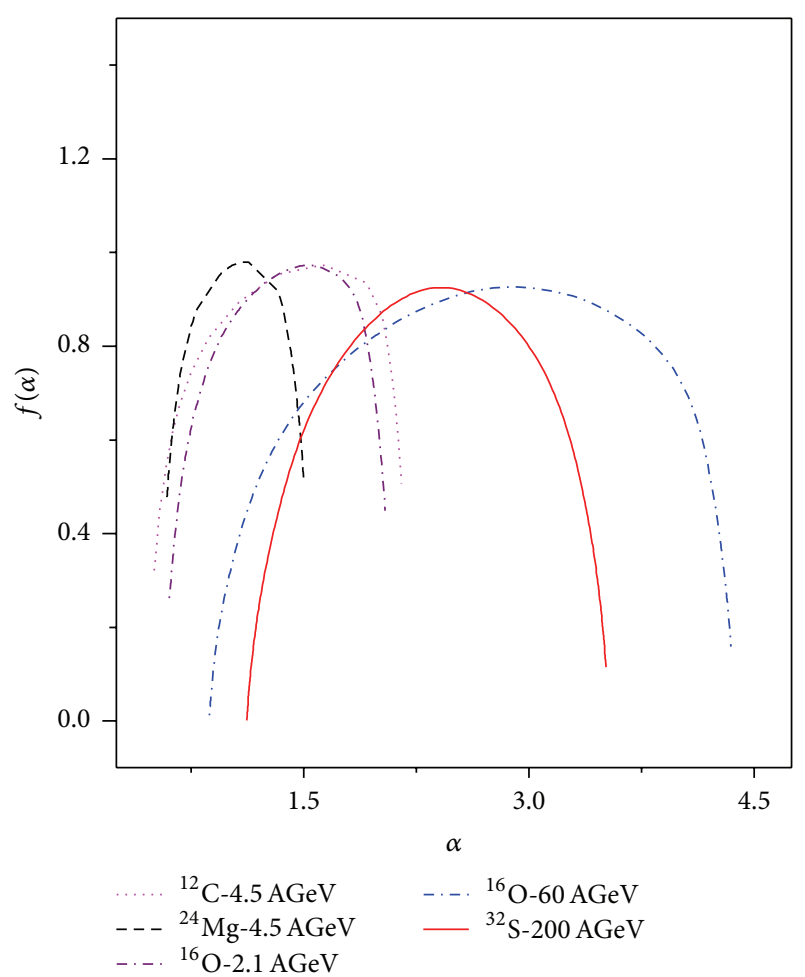

FIGURE 6: Multifractal spectrum for the considered interactions.

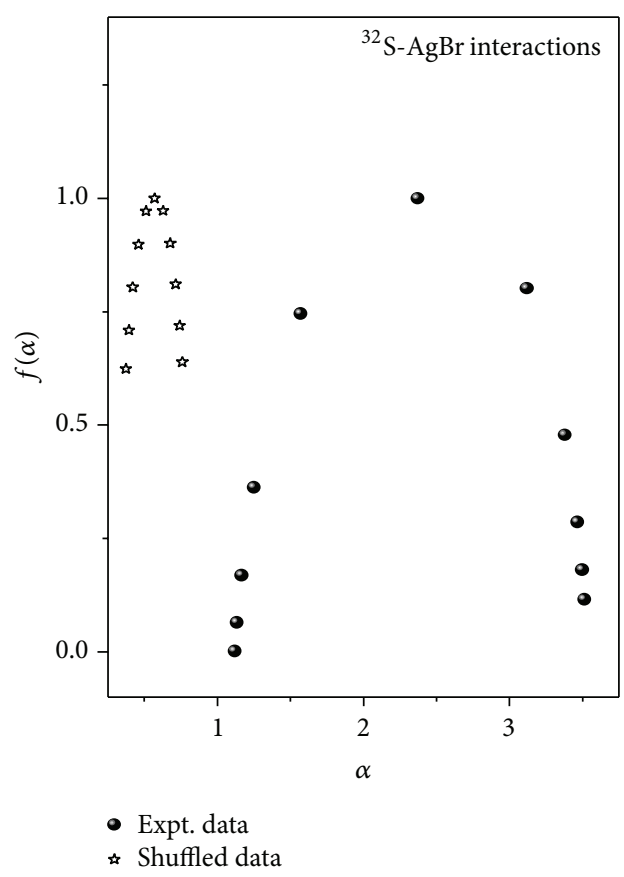

FIGURE 7: Comparison of multifractal spectrum for experimental and shuffled data for ${ }^{32} \mathrm{~S}-\mathrm{AgBr}$ interactions at $200 \mathrm{AGeV}$.

(iv) If projectile mass and energy simultaneously change in the same manner, that is, either both increase or both decrease, the strength of multifractality is predominantly affected by the mass of the projectile if energy is of the same order. This fact is revealed if we consider the pair of interactions (i) ${ }^{16} \mathrm{O}-\mathrm{AgBr}$ at $2.1 \mathrm{AGeV}$ and ${ }^{24} \mathrm{Mg}-\mathrm{AgBr}$ at $4.5 \mathrm{AGeV}$ and (ii) ${ }^{16} \mathrm{O}$ $\mathrm{AgBr}$ at $60 \mathrm{AGeV}$ and ${ }^{32} \mathrm{~S}-\mathrm{AgBr}$ at $200 \mathrm{AGeV}$. On the other hand, if mass difference is little, strength of multifractality is mainly determined by the energy difference of the projectile. This is demonstrated by the interactions ${ }^{12} \mathrm{C}-\mathrm{AgBr}$ at $4.5 \mathrm{AGeV}$ and ${ }^{16} \mathrm{O}-\mathrm{AgBr}$ at $60 \mathrm{AGeV}$ pair.

(v) Finally, if we go from relativistic energy regime to the ultrarelativistic one, influence of energy is much more prominent compared to mass. This is noticed comparing ${ }^{16} \mathrm{O}-\mathrm{AgBr}$ at $2.1 \mathrm{AGeV},{ }^{24} \mathrm{Mg}-\mathrm{AgBr}$ at $4.5 \mathrm{AGeV}$, and ${ }^{12} \mathrm{C}-\mathrm{AgBr}$ at $4.5 \mathrm{AGeV}$ interactions with the ${ }^{32} \mathrm{~S}$ $\mathrm{AgBr}$ interactions at $200 \mathrm{AGeV}$.

\section{Conclusions}

We have presented a systematic study on pion density fluctuation in pseudorapidity spectra in the framework of sophisticated MFDFA technique using various heavy ion projectiles covering a wide range of energy starting from a few $\mathrm{GeV}$ to a few hundred $\mathrm{GeV}$. The main observations of the analysis may be summarized as follows:

(i) Both $h(q)$ and $\tau(q)$ spectra speak in favor of multifractal pion density fluctuation in pseudorapidity space for all the considered A-A interactions.

(ii) Multifractal parameters $h_{q}$ and $\tau_{q}$ and the multifractal spectrum for the shuffled data clearly demonstrate that the observed multifractality of the experimental data does not originate from the trivial broad probability density distribution but occurs due to dynamics.

(iii) The study suggests multifractal pion production dynamics in heavy ion interactions.

(iv) The strength of multifractality is influenced both by mass and by energy of the projectile beam.

(v) For the same projectile beam multifractality increases with projectile energy. On the other hand, it decreases with mass of the projectile beams having the same energy.

(vi) When both mass and energy change, effect of mass is dominant if their energy does not differ much. But multifractality is much more influenced by energy of the projectile beam if mass difference is small.

(vii) From the value of ordinary Hurst exponent we can conclude that long range correlation is present among the pions for all of the considered heavy ion interactions irrespective of projectile mass and energy.

\section{Competing Interests}

The authors declare that they have no competing interests. 


\section{Acknowledgments}

The authors are grateful to Professor P. L. Jain, State University of Buffalo, Buffalo, USA, Professor P. S. Young of Mississippi State University, Mississippi, and Professor K. D. Tolstov, JINR, Dubna, U.S.S.R., for providing them with exposed and developed emulsion plates used for this analysis. One of the authors, Miss Gopa Bhoumik, acknowledges CSIR for a Junior Research Fellowship (Sanction no. 09/096(0802)/2013-EMR-I) during this work.

\section{References}

[1] B. B. Mandelbrot, The Fractal Geometry of Nature, Freeman, San Francisco, Calif, USA, 1999.

[2] S. Ahmad and M. A. Ahmad, "A comparative study of multifractal moments in relativistic heavy-ion collisions," Journal of Physics G: Nuclear and Particle Physics, vol. 32, no. 9, pp. 12791293, 2006.

[3] M. M. Khan, N. Ahmad, A. Kamal, A. A. Masoodi, and M. Irfan, "Correlation and fluctuations in relativistic nucleus-nucleus collisions," Indian Journal of Physics, vol. 85, no. 1, pp. 189-193, 2011.

[4] G. Singh and P. L. Jain, "Multifractal analysis of ${ }^{197} \mathrm{Au}$ collisions at 10.6A GeV," Physical Review C, vol. 50, no. 5, pp. 2508-2515, 1994.

[5] A. Bershadskii, "Multifractal critical chaos and intermittency in multiparticle production," Journal of Physics G: Nuclear and Particle Physics, vol. 26, no. 7, pp. 1011-1015, 2000.

[6] Z. Chen, P. Ch. Ivanov, K. Hu, and H. E. Stanley, "Effect of nonstationarities on detrended fluctuation analysis," Physical Review E, vol. 65, no. 4, pp. 041107-041122, 2002.

[7] S. V. Chekanov and V. I. Kuvshinov, "Multifractal multiplicity distribution in bunching parameter analysis," Journal of Physics G: Nuclear and Particle Physics, vol. 22, no. 5, pp. 601-610, 1996.

[8] A. Bialas, "Intermittency '90," Nuclear Physics A, vol. 525, pp. 345-360, 1991.

[9] R. Peschanski, "Intermittency in particle collisions," International Journal of Modern Physics A, vol. 6, no. 21, pp. 3681-3722, 1991.

[10] E. A. De Wolf, I. M. Dremin, and W. Kittel, "Scaling laws for density correlations and fluctuations in multiparticle dynamics," Physics Reports, vol. 270, no. 1-2, pp. 1-141, 1996.

[11] P. Carruthers and M. Duong-Van, "Evidence for a common fractal dimension in multiparticle production," Los Alamos Report LA-UR-83-2419, 1983.

[12] R. C. Hwa, "Fractal measures in multiparticle production," Physical Review D, vol. 41, no. 5, pp. 1456-1462, 1990.

[13] R. C. Hwa and J. Pan, "Fractal behavior of multiplicity fluctuations in high-energy collisions," Physical Review D, vol. 45, no. 5, pp. 1476-1483, 1992.

[14] I. M. Dermin, "The fractal correlation measure for multiple production," Modern Physics Letters A, vol. 3, no. 14, p. 1333, 1988.

[15] P. Carruthers, "Fractal structures and correlations in hadronic multiparticle distributions," International Journal of Modern Physics A, vol. 4, no. 20, pp. 5587-5614, 1989.

[16] Ph. Brax and R. Peschanski, "Multifractal analysis of intermittency and phase transitions in multiparticle dynamics," Nuclear Physics B, vol. 346, no. 1, pp. 65-83, 1990.
[17] F. Takagi, "Multifractal structure of multiplicity distributions in particle collisions at high energies," Physical Review Letters, vol. 72, no. 1, pp. 32-35, 1994.

[18] G. Paladin and A. Vulpiani, "Anomalous scaling laws in multifractal objects," Physics Reports, vol. 156, no. 4, pp. 147-225, 1987.

[19] P. Grassberger and I. Procaccia, "Dimensions and entropies of strange attractors from a fluctuating dynamics approach," Physica D: Nonlinear Phenomena, vol. 13, no. 1-2, pp. 34-54, 1984.

[20] T. C. Halsey, M. H. Jensen, L. P. Kadanoff, I. Procaccia, and B. I. Shraiman, "Fractal measures and their singularities: the characterization of strange sets," Physical Review A, vol. 33, no. 2, pp. 1141-1151, 1986.

[21] M. I. Haque, M. Tariq, and T. Hussain, "Presence of multifractality in high-energy nuclear collisions," Journal of Modern Physics, vol. 5, no. 17, pp. 1889-1895, 2014.

[22] N. Ahmad, A. Kamal, M. M. Khan, Hushnud, and A. Tufail, "A study of multifractal spectra and renyi dimensions in 14.5A $\mathrm{GeV} / \mathrm{c}^{28}$ Si-nucleus collisions," Journal of Modern Physics, vol. 5, pp. 1288-1293, 2014.

[23] D. Ghosh, A. Deb, R. Chattopadhyay et al., "Evidence of multifractal nature of target-evaporated slow particles produced in ultrarelativistic heavy ion interactions," Physical Review C, vol. 58, no. 6, p. 3553, 1998.

[24] C.-K. Peng, S. V. Buldyrev, S. Havlin, M. Simons, H. E. Stanley, and A. L. Goldberger, "Mosaic organization of DNA nucleotides," Physical Review E, vol. 49, no. 2, pp. 1685-1689, 1994.

[25] J. W. Kantelhardt, D. Rybski, S. A. Zschiegner et al., "Multifractality of river runoff and precipitation: comparison of fluctuation analysis and wavelet methods," Physica A: Statistical Mechanics and Its Applications, vol. 330, no. 1-2, pp. 240-245, 2003.

[26] I. Daubechies, Ten Lectures on Wavelets, SIAM, Philadelphia, Pa, USA, 1992.

[27] S. Mallat, A Wavelet Tour of Signal Processing, Academic Press, 1999.

[28] P. Manimaran, P. K. Panigrahi, and J. C. Parikh, "Wavelet analysis and scaling properties of time series," Physical Review E, vol. 72, Article ID 046120, 2005.

[29] P. Manimaran, P. Anantha Lakshmi, and P. K. Panigrahi, "Spectral fluctuation characterization of random matrix ensembles through wavelets," Journal of Physics A: Mathematical and General, vol. 39, no. 42, p. L599, 2006.

[30] J. W. Kantelhardt, S. A. Zschiegner, E. Koscielny-Bunde, S. Havlin, A. Bunde, and H. E. Stanley, "Multifractal detrended fluctuation analysis of nonstationary time series," Physica A: Statistical Mechanics and its Applications, vol. 316, no. 1-4, pp. 87-114, 2002.

[31] M. S. Taqqu, V. Teverovsky, and W. Willinger, "Estimators for long-range dependence: an empirical study," Fractals, vol. 3, no. 4, pp. 785-798, 1995.

[32] J. P. Bouchaud, M. Potters, and M. Meyer, "Apparent multifractality in financial time series," The European Physical Journal B, vol. 13, no. 3, pp. 595-599, 2000.

[33] P. Mali and A. Mukhopadhyay, "Multifractal characterization of gold market: a multifractal detrended fluctuation analysis," Physica A: Statistical Mechanics and Its Applications, vol. 413, pp. 361-372, 2014.

[34] Y. Yuan, X.-T. Zhuang, and X. Jin, "Measuring multifractality of stock price fluctuation using multifractal detrended fluctuation 
analysis," Physica A: Statistical Mechanics and Its Applications, vol. 388, no. 11, pp. 2189-2197, 2009.

[35] Y. Wang, L. Liu, and R. Gu, "Analysis of efficiency for Shenzhen stock market based on multifractal detrended fluctuation analysis," International Review of Financial Analysis, vol. 18, no. 5, pp. 271-276, 2009.

[36] P. Norouzzadeh and B. Rahmani, "A multifractal detrended fluctuation description of Iranian rial-US dollar exchange rate," Physica A: Statistical Mechanics and Its Applications, vol. 367, pp. 328-336, 2006.

[37] P. Zhou, F. Li, W.-Y. Liu, and M. Yang, World Congress on Medical Physics and Biomedical Engineering, Edited by S. I. Kim, T. S. Suh, Springer, Berlin, Germany, 2007.

[38] S. Dutta, D. Ghosh, and S. Chatterjee, "Multifractal detrended fluctuation analysis of human gait diseases," Frontiers in Physiology, vol. 4, article 274, 2013.

[39] P. Mali, "Multifractal characterization of global temperature anomalies," Theoretical and Applied Climatology, vol. 121, no. 34, pp. 641-648, 2015.

[40] R. B. de Benicio, T. Stošić, P. H. de Figueirêdo, and B. D. Stošić, "Multifractal behavior of wild-land and forest fire time series in Brazil," Physica A: Statistical Mechanics and Its Applications, vol. 392, no. 24, pp. 6367-6374, 2013.

[41] J. W. Kantelhardt, A. Bunde, and L. Schweitzer, "Are the phases in the Anderson model long-range correlated?" Physica A, vol. 266, pp. 461-464, 1999.

[42] N. Vandewalle, M. Ausloos, M. Houssa, P. W. Mertens, and M. M. Heyns, "Non-Gaussian behavior and anticorrelations in ultrathin gate oxides after soft breakdown," Applied Physics Letters, vol. 74, no. 11, pp. 1579-1581, 1999.

[43] Y. X. Zhang, W. Y. Qian, and C. B. Yang, "Multifractal structure of pseudorapidity and azimuthal distributions of the shower particles in $\mathrm{Au}+\mathrm{Au}$ collisions at $200 \mathrm{~A} \mathrm{GeV}$,' International Journal of Modern Physics A, vol. 23, no. 18, p. 2809, 2008.

[44] X. Wang and C. B. Yang, "Fractal properties of particles in phase space from URQMD model," International Journal of Modern Physics E, vol. 22, no. 4, Article ID 1350021, 8 pages, 2013.

[45] P. Mali, S. Sarkar, S. Ghosh, A. Mukhopadhyay, and G. Singh, "Multifractal detrended fluctuation analysis of particle density fluctuations in high-energy nuclear collisions," Physica A: Statistical Mechanics and Its Applications, vol. 424, pp. 25-33, 2015.

[46] D. Ghosh, P. Ghosh, A. Ghosh, and J. Roy, "Intermittency and fragmentation of target residue in high-energy nuclear interactions," Physical Review C, vol. 49, no. 4, pp. R1747-R1750, 1994.

[47] D. Ghosh, A. Mukhopadhyay, A. Ghosh, and J. Roy, "Zonal poissonian pion multiplicity in central ${ }^{24} \mathrm{Mg}-\mathrm{AgBr}$ collisions at dubna energy," Modern Physics Letters A, vol. 4, no. 13, pp. 1197$1202,1989$.

[48] D. Ghosh, J. Roy, and R. Sengupta, "Study of multiparticle production in the interaction of ${ }^{12} \mathrm{C}$ with photoemulsion nuclei at $4.5 \mathrm{GeV} / \mathrm{c}$ per nucleon," Nuclear Physics A, vol. 468, no. 3-4, pp. 719-738, 1987.

[49] K. Sengupta, P. L. Jain, G. Singh, and S. N. Kim, "Intermittency in multiparticle production at ultra-relativistic heavy ion collisions," Physics Letters B, vol. 236, no. 2, pp. 219-223, 1990.

[50] C. F. Powell et al., Pergamon, Oxford, UK, pp. 450-464, 1959.

[51] D. Ghosh, P. Ghosh, A. Ghosh, and J. Roy, "Nonstatistical fluctuations in fragmentation of target nuclei in high energy nuclear interactions," Journal of Physics G: Nuclear and Particle Physics, vol. 20, no. 7, article 1077, 1994.
[52] D. Ghosh, B. Biswas, A. Deb, and J. Roy, "Evidence of scaling of void probability in nucleus-nucleus interactions at few $\mathrm{GeV}$ energy," Physical Review C, vol. 56, no. 5, pp. 2879-2881, 1997.

[53] D. Ghosh, A. K. Jafry, A. Deb, S. Sarkar, R. Chattopadhyay, and S. Das, "Multidimensional intermittency analysis in ultrarelativistic heavy ion interaction," Physical Review C, vol. 59, no. 4, p. 2286, 1999.

[54] D. Ghosh, M. Lahiri, A. Deb et al., "Factorial correlator study in ${ }^{32} \mathrm{Ag} / \mathrm{Br}$ interaction at $200 \mathrm{~A} \mathrm{GeV,"} \mathrm{Physical} \mathrm{Review} \mathrm{C,} \mathrm{vol.} \mathrm{52,}$ no. 4, p. 2092, 1995.

[55] J. Feder, Fractals, Plenum Press, New York, NY, USA, 1988.

[56] H.-O. Peitgen, H. Jürgens, and D. Saupe, Chaos and Fractals, Appendix B, Springer, New York, NY, USA, 1992.

[57] Y. Ashkenazy, D. R. Baker, H. Gildor, and S. Havlin, "Nonlinearity and multifractality of climate change in the past 420,000 years," Geophysical Research Letters, vol. 30, no. 22, p. 2146, 2003.

[58] Y. U. Shimizu, S. Thurner, and K. Ehrenberger, "Multifractal spectra as a measure of complexity in human posture," Fractals, vol. 10, no. 1, pp. 103-116, 2002.

[59] L. Telesca, M. Balasco, G. Colangelo, V. Lapenna, and M. Macchiato, "Investigating the multifractal properties of geoelectrical signals measured in southern Italy," Physics and Chemistry of the Earth, vol. 29, no. 4-9, pp. 295-303, 2004.

[60] C. Barman, H. Chaudhuri, A. Deb, D. Ghose, and B. Sinha, "The essence of multifractal detrended fluctuation technique to explore the dynamics of soil radon precursor for earthquakes," Natural Hazards, vol. 78, no. 2, pp. 855-877, 2015. 

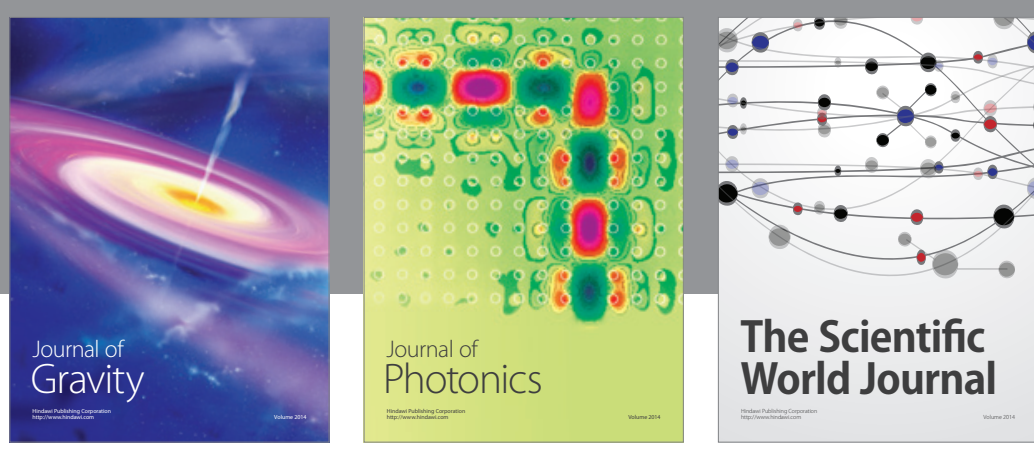

The Scientific World Journal
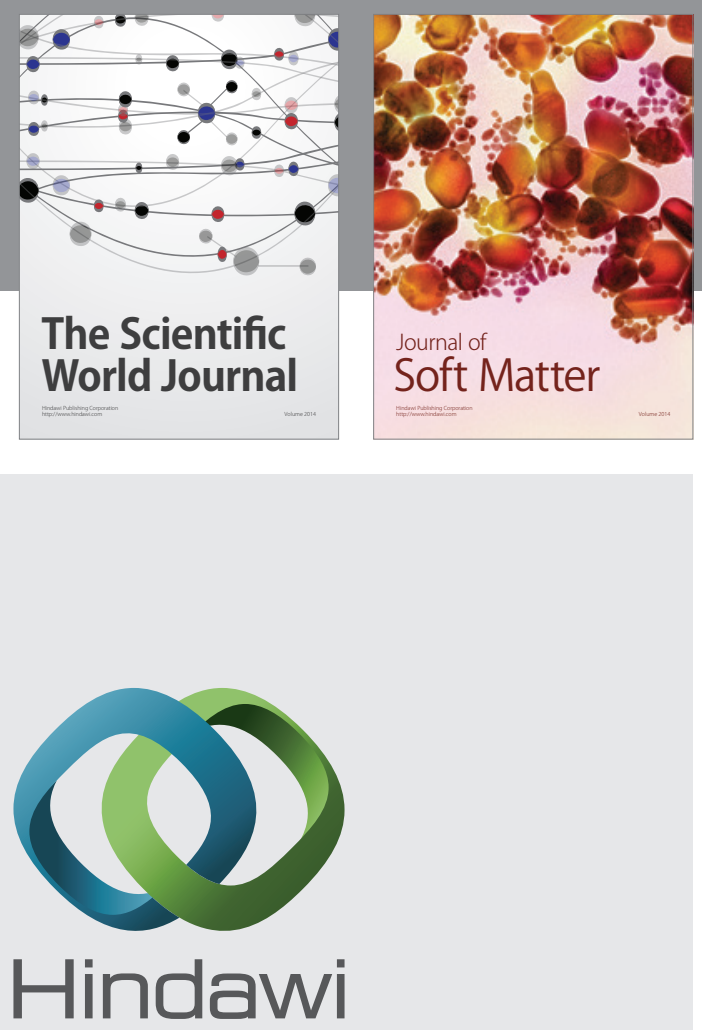

Submit your manuscripts at

http://www.hindawi.com

nternational Journal of

Statistical Mechanics
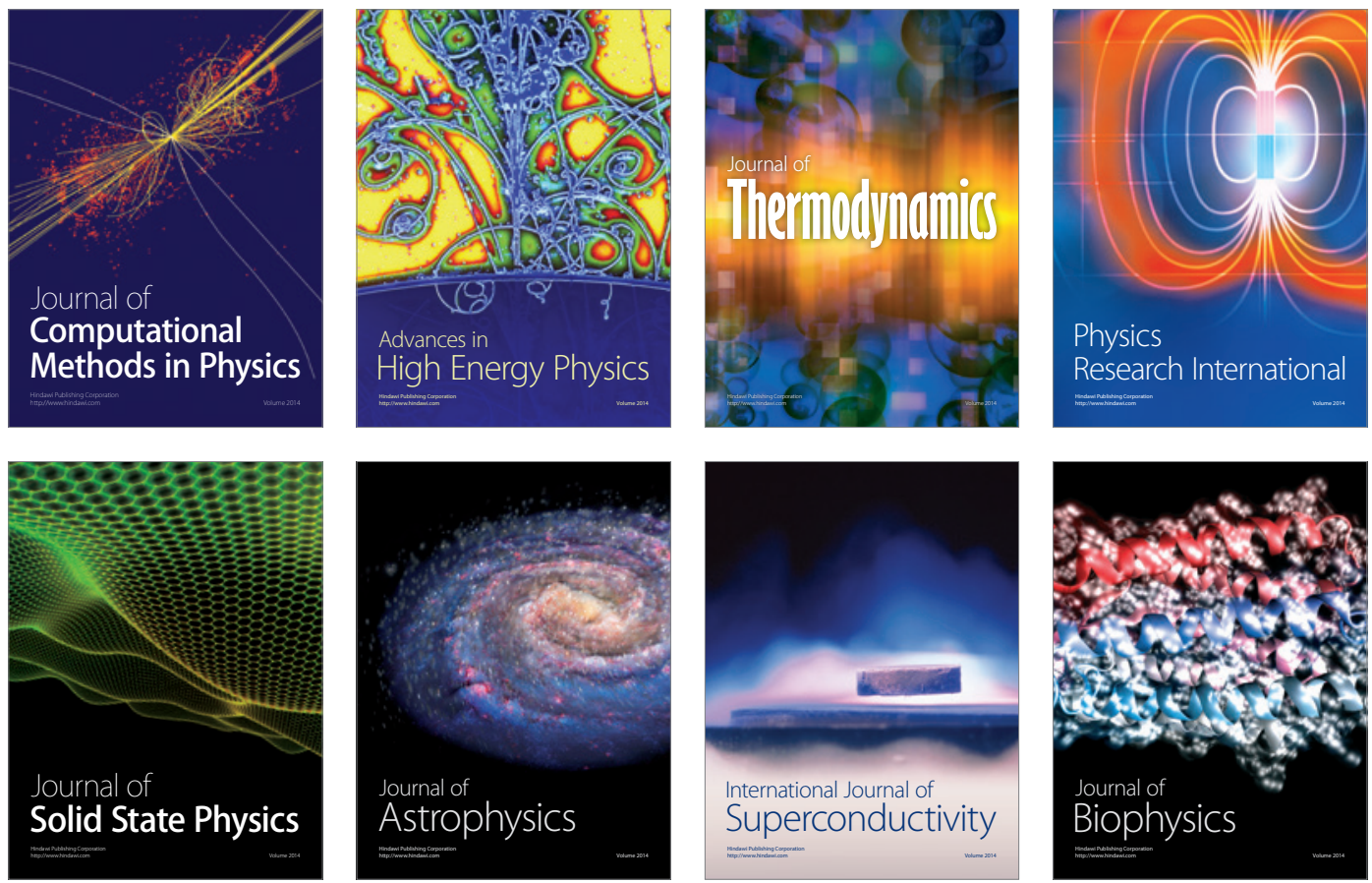
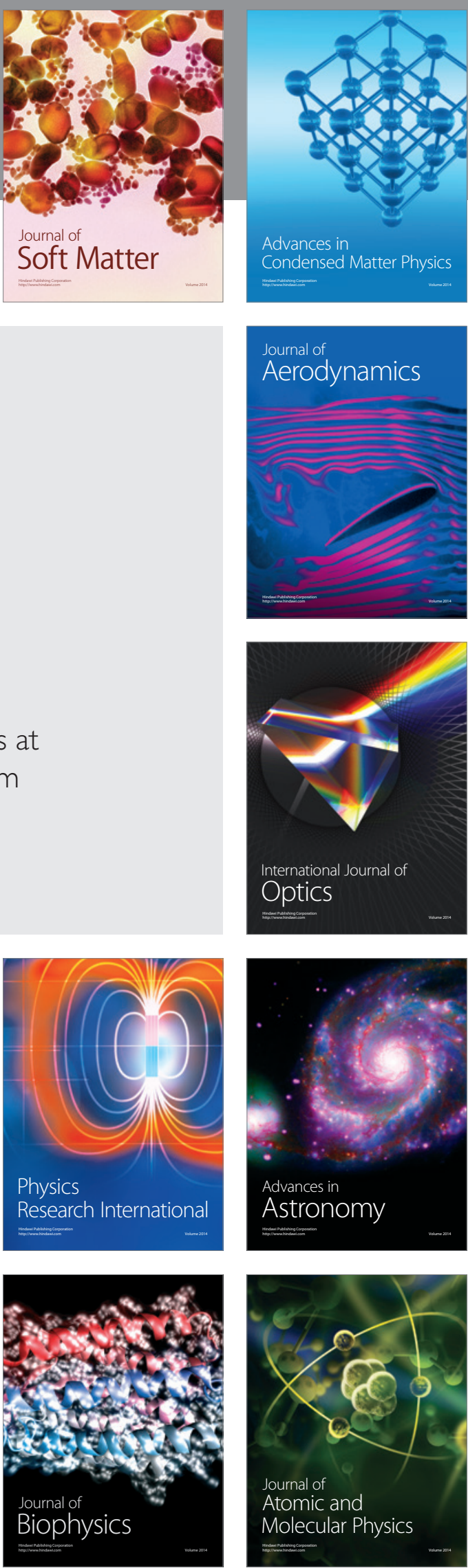